\title{
Carillas directas con resinas compuestas: una alternativa en Operatoria Dental
}

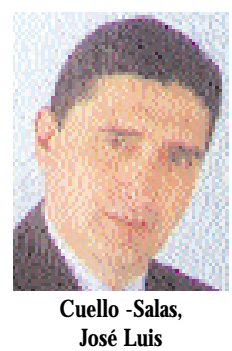

\section{Composite resin direct veneers: an alternative in Operative Dentistry}

Cuello-Salas, José Luis*

Pasquini-Comba, Miriam**

Bazáez- Frete, Mónica**

Oiva-Bazáez, Constanza**

*Doctor en Odontología. Ex-profesor encargado Cátedra de Operatoria Dental \| «B». Universidad Nacional de Córdoba. Argentina. Docente Universitario Área de Rehabilitación Bucal: Operatoria Dental. Miembro de la Comisión Científica del Colegio Odontológico de Córdoba. Argentina.

**Miembros del Equipo de Trabajo
Resumen: Orientándonos hacia una odontología preventiva y de tecnología adhesiva, es importante recordar el rol que cumple la estética y la conservación de las estructuras anatómicas naturales del elemento dentario. Para ello, las restauraciones en el sector anterior con materiales estéticos como las resinas compuestas fotopolimerizables, despertaron interés y una gran demanda, debido a las ventajas que ofrecen dichos materiales. Por lo tanto, se presenta al odontólogo general, una restauración directa y semipermanente, como la confección de carillas directas con resinas compuestas, siendo una alternativa en operatoria dental a la clásica corona total, para solucionar las distintas alteraciones que se presentan en las caras vestibulares de los elementos dentarios anteriores.

Palabras Claves: Facetas, Carillas, Resinas compuestas.

Abstract: Tending towards a preventive dentistry and the use of adhesive technologies, it is important to remember the role of aesthetics and the conservation of the natural anatomical structures of the tooth. Therefore, restorations in the anterior region performed with aesthetic materials such as light-cured composite resins promoted interest and a large demand, due to the advantages offered by these materials. For this reason, a direct and semi-permanent restorative option, composite resin direct veneers, is shown to the general dentist, being an alternative in operative dentistry to the classical full-coverage crown in solving the different alterations present on the buccal surfaces of anterior teeth.

Key Words: Facings, Venners, Composite resins.

\begin{tabular}{ccc}
\hline Fecha recepción & Fecha última revisión & Fecha aceptación \\
$23-08-2002$ & $13-03-2003$ & $28-05-2003$ \\
\hline
\end{tabular}

BIBUD [1138-123X (2003)8:4; julio-agosto 349-468]

Cuello-Salas J L, Pasquini-Comba M, Bazáez-Frete M, Oliva-Bazáez C. Carillas directas con resinas compuestas: una alternativa en Operatoria Dental. RCOE 2003;8(4):415-421. 


\section{Introducción}

Actualmente, nadie duda que la demanda en los tratamientos odontológicos estéticos ha aumentado, pudiéndose observar como los pacientes muestran mayor interés, no solo por mejorar su aspecto funcional sino también el estético.

En el sector anterior, además de las distintas restauraciones convencionales que se pueden realizar a través de la tecnología adhesiva, se pueden solucionar otros casos clínicos, donde el objetivo final es conservar la estructura dentaria natural sana y lograr estética'.

Durante mucho tiempo, para mejorar los aspectos estéticos, se realizaban restauraciones de coronas totales, que es un sistema mas mutilante e invasivo, para el cual es necesario eliminar gran cantidad de tejido sano $^{2}$. A medida que ha ido transcurriendo el tiempo, diversos autores como Simonsen y Calamia ${ }^{3}, \mathrm{Horn}^{4}$ entre otros, trataron de solucionar este inconveniente, proponiendo distintas alternativas.

Por supuesto, el desafío es mayor y sin interferir con otro perfil como es la prótesis fija convencional, se puede lograr solucionar distintas alteraciones que se presentan en la cara vestibular de los elementos dentarios anteriores, a través de restauraciones adhesivas de carillas directas con resinas compuestas, siendo estas una alternativa semipermanente a la clásica corona total, además de ser menos invasiva y fundamentalmente, por eliminar menor cantidad de tejido dentario sano.

El objetivo de este trabajo es presentar al odontólogo general, la técnica de confección de carillas directas con resinas compuestas, sus indica-

\section{Tabla L Clasificación de las carillas}

\begin{tabular}{l|l} 
Según el material & Según el método \\
\hline $\begin{array}{l}\text { Resinas } \\
\text { Porcelanas } \\
\text { Otros (Cerómeros) }\end{array}$ & Resinas compuestas $\longrightarrow$ Directa (mano alzada) \\
& Porcelanas Indirecta (en modelo) \\
\end{tabular}

ciones, contraindicaciones, ventajas y desventajas, como así también, su importancia para solucionar las distintas alteraciones que se presentan en las caras vestibulares de elementos dentarios anteriores.

\section{Clasificación de las carillas}

Según Macchi, $\mathrm{R}^{5}$, carilla es un «bloque que se fija a la superficie vestibular de un diente anterior, fundamentalmente para mejorar sus aspectos estéticos» (tabla 1).

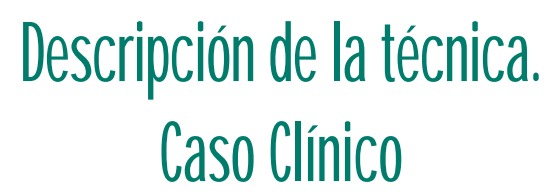

Concurre a la consulta una paciente de sexo femenino, 23 años de edad, procedente de la ciudad de Bell Ville, Provincia de Córdoba, Argentina, por razones estéticas.

La paciente manifiesta, la imposibilidad de sonreír por las manchas que presentan sus incisivos centrales superiores (figs. 1,2 ).

Primeramente, se confeccionó una historia clínica y una odontológica, que además de la importancia legal, nos permite conocer al paciente reco- giendo datos, para luego, determinar el diagnóstico, pronóstico y plan de tratamiento.

Posteriormente, es necesario evaluar el estado de salud periodontal, que debe ser bueno, para realizar cualquier restauración adhesiva en operatoria dental.

Se procede a efectuar el diagnóstico, pronóstico y plan de tratamiento donde se tuvo en cuenta los siguiente elementos:

Para el diagnóstico: estado de salud pulpar, radiografías previas y diagnóstico diferencial.

Se evalúa el estado de salud pulpar de los elementos a tratar, se procede a la toma de radiografías previas y posteriormente se realiza, el diagnóstico diferencial, entre las alteraciones de color más frecuentes que se presentan en el sector anterior, tales como, hipoplasia, hipocalcificación, caries, restauraciones estéticas decoloreadas, amelogénesis imperfecta y fluorosis ${ }^{6,7}$.

Luego de evaluar algunos elementos que las diferencian unas de otras como la etiología, dentición, forma, color, localización, tejidos afectados, textura superficial e inspección clínica, se determinó que la alteración cromática era por fluorosis, ocasionada por un exceso de flúor en las aguas de bebidas característica de zonas 


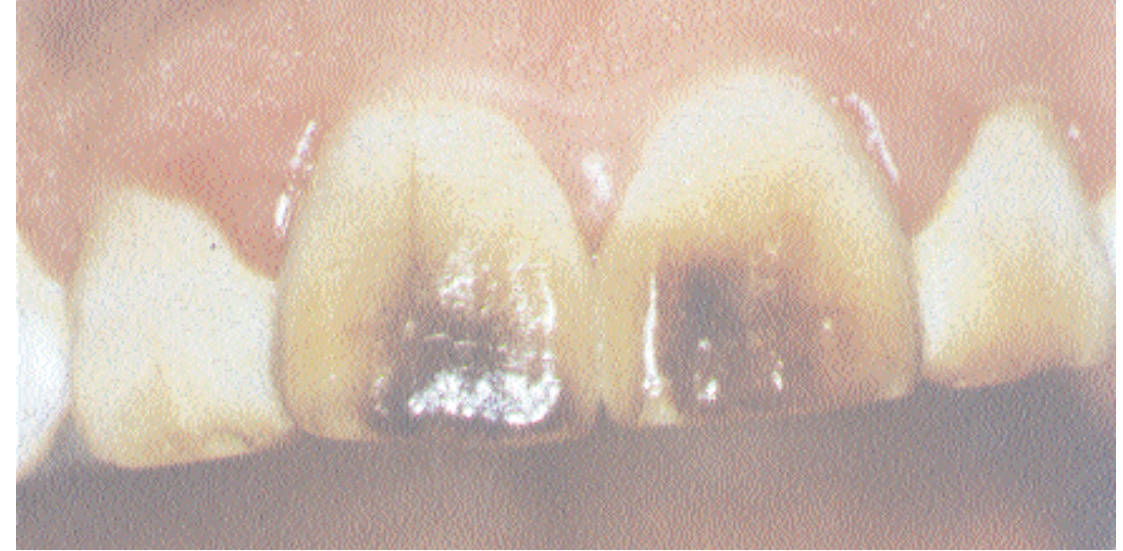

Figura 1: Vista vestibular de los dientes 11 y 21 con manchas.

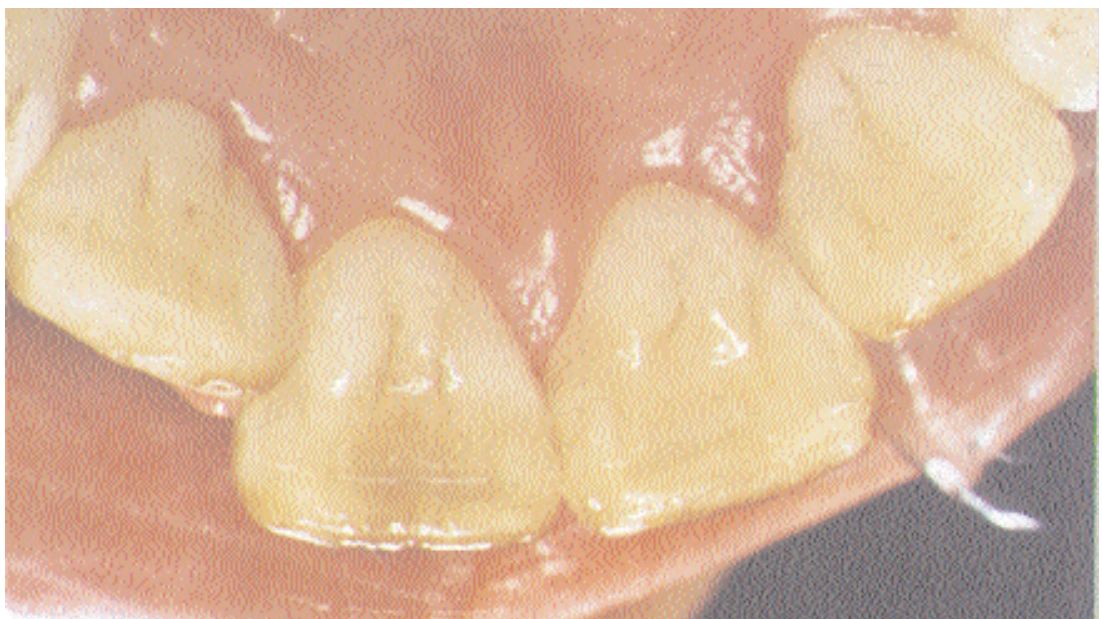

Figura 2: Vista palatina de los dientes 11 y 21 sin invasión de manchas.

endémicas, como Bell Ville, al sur de la Provincia de Córdoba, Argentina, procedencia de la paciente ${ }^{6,7}$.

Para el plan de tratamiento: evaluación del esmalte, análisis de la oclusión, confección de modelo de estudio y fotografías previas.

Con respecto a la evaluación del esmalte remanente, debe ser suficiente en cantidad y calidad, para efectuar este tipo de restauraciones adhesivas directas.

Se debe realizar el análisis de la oclusión, ya que las mordidas borde a borde o los hábitos parafuncionales, son desfavorables, para la confección de carillas directas con resinas compuestas.

Se recomienda, confeccionar un modelo de estudio, para control de la forma, contorno y tamaño de los dientes a tratar. Por último, se debe documentar el caso a realizar, previamente, con fotografías de color, para presentar al paciente el resultado alcanzado, además del valor legal que presenta.

El plan de tratamiento, se dividió en dos etapas: la primera, consistió en realizar, blanqueamiento dental am- bulatorio ${ }^{8-10^{* *-11 *}}$, con la finalidad de disminuir la tonalidad de las manchas, de tal manera, evitar destruir la menor cantidad de tejido dentario sano.

Después de cuatro semanas de tratamiento, se decide pasar a la segunda etapa del tratamiento, mediante la confección de carillas directas con resinas compuestas ${ }^{10^{* *}, 11^{*-15}}$ (figs. 3, 4).

\section{Maniobras operatorias clínicas}

L primero que se realiza, es la toma del color, los registros de los contactos oclusales y posteriormente, se efectúa la técnica de anestesia para trabajar con mayor comodidad.

La siguiente maniobra, es el aislamiento del campo operatorio, ya que durante la preparación vestibular del diente a tratar, es conveniente utilizar aislamiento relativo, puesto que el absoluto con dique de goma, perjudica la visión natural y dificulta las caracterizaciones de los elementos dentarios a tratar.

\section{Preparación vestibular del elemento dentario}

Se protegen los dientes vecinos, con bandas metálicas o algún otro dispositivo que evite dañar las estructuras dentarias sanas; luego, se reduce el esmalte vestibular, aproximadamente $0,4 \mathrm{~mm}$ en la región gingival y $0,5 \mathrm{~mm}$ en el tercio medio e incisal, en elementos dentarios con una discreta alteración de color, sin sobrepasar el borde incisal hacia palatino.

Para obtener, una reducción homogénea, se recomienda utilizar una piedra de diamante esférica 011, crean- 


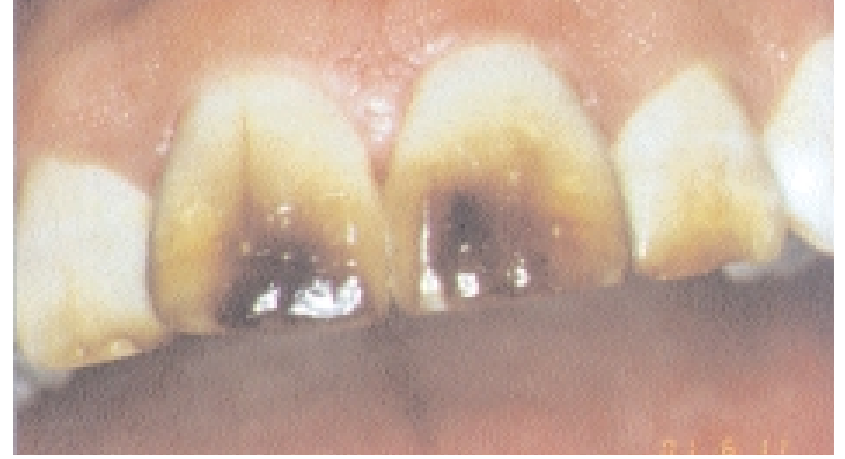

Figura 3: Fotografia previa mostrando el buen estado periodontal.

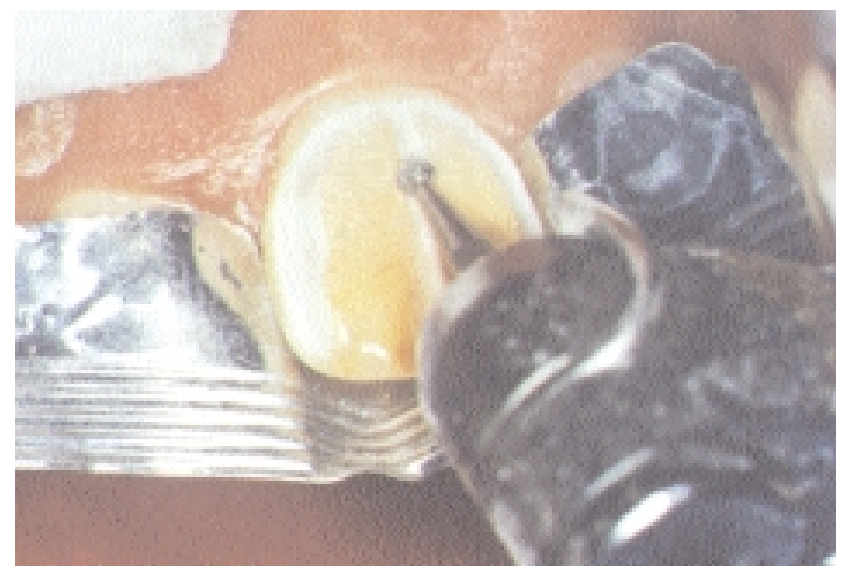

Figura 5: Creación de surcos con instrumental rotatorio, para estandarizar la profundidad de la preparación vestibular

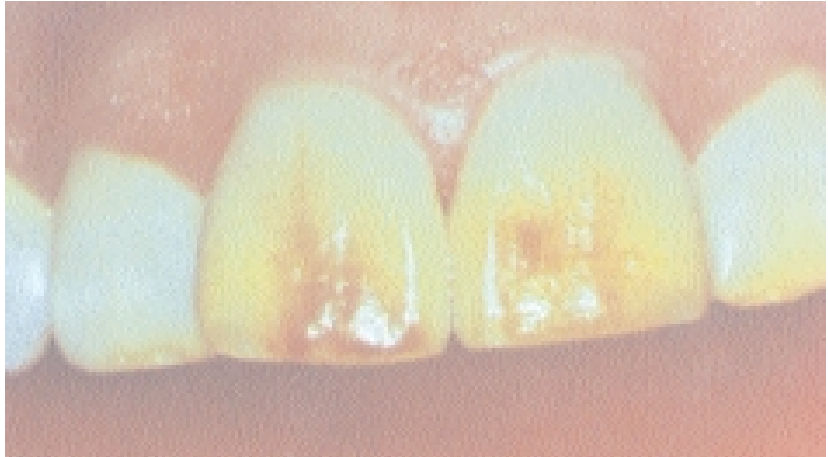

Figura 4: Fotografia posterior a la técnica de blanqueamiento ambulatorio.

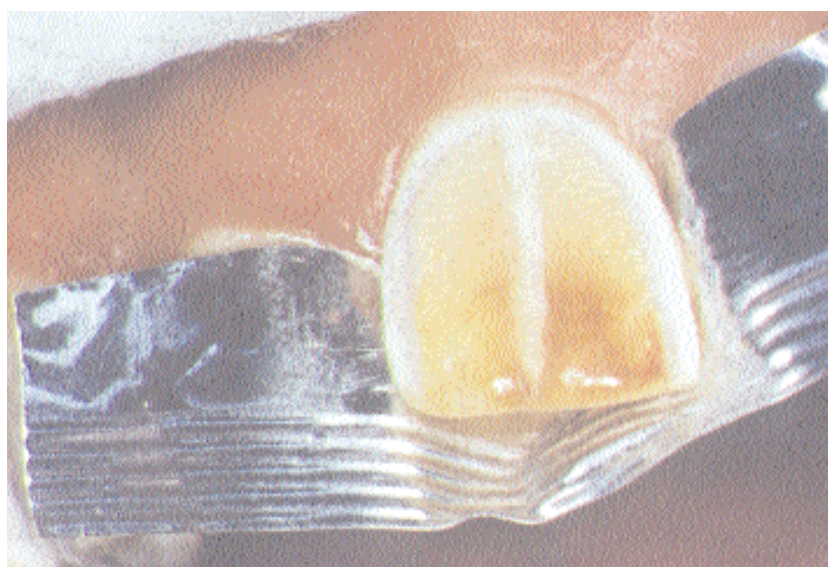

Figura 6: Vista vestibular de los surcos de orientación

do ranuras o surcos, que sirven de orientación para estandarizar, posteriormente, la profundidad requerida (figs. 5, 6).

Luego, se unen las ranuras o surcos con una piedra de diamante troncocónica 011 de punta redondeada, respetando la curvatura original de la superficie vestibular del elemento dentario, tanto en sentido gingivoincisal como proximo-proximal. Es importante, que las líneas de terminación gingival y proximal, sean redondeadas o en chanfer (fig. 7).

Posteriormente, se realiza la limpieza de la preparación, para luego, efectuar el aislamiento absoluto del

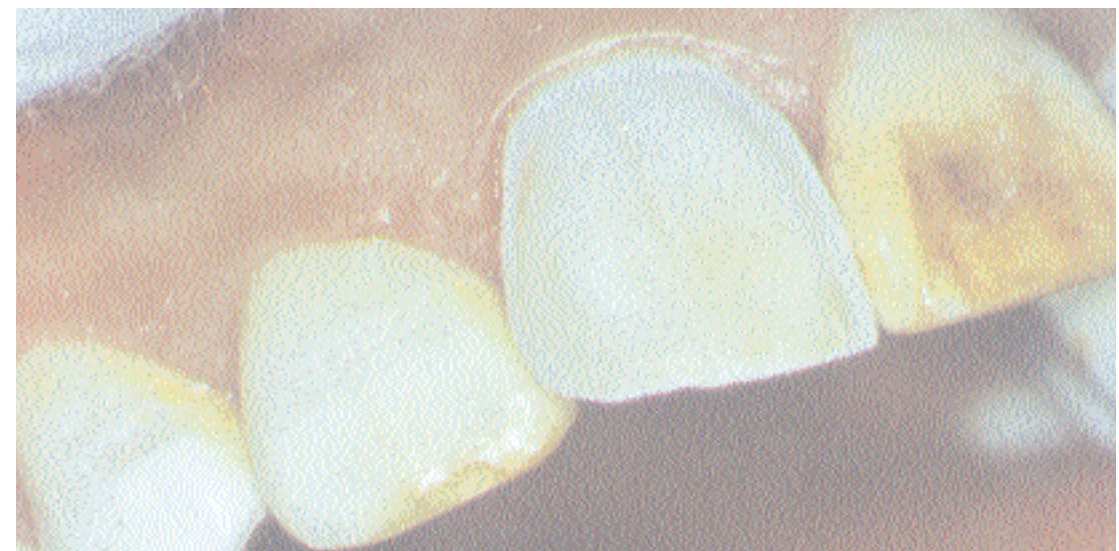

Figura 7: Preparación vestibular terminada.

campo operatorio, colocando un retractor gingival de Hatch para retraer la encía, como así también, la goma del dique, permitiendo lograr una buena visualización de los límites de la preparación (fig. 8).

RCOE, 2003, Vol 8, №4, 415-421 


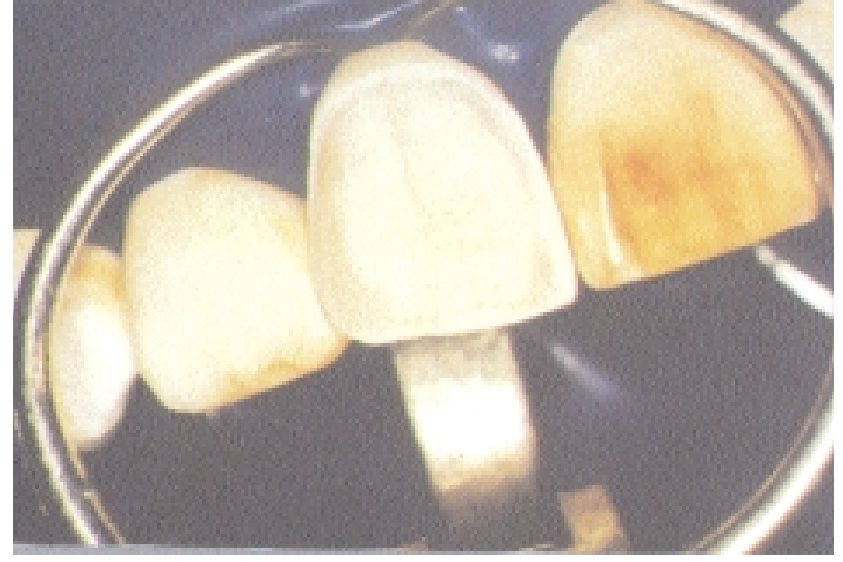

Figura 8: Retractor gingival de Hatch para retraer la encía y goma del dique.

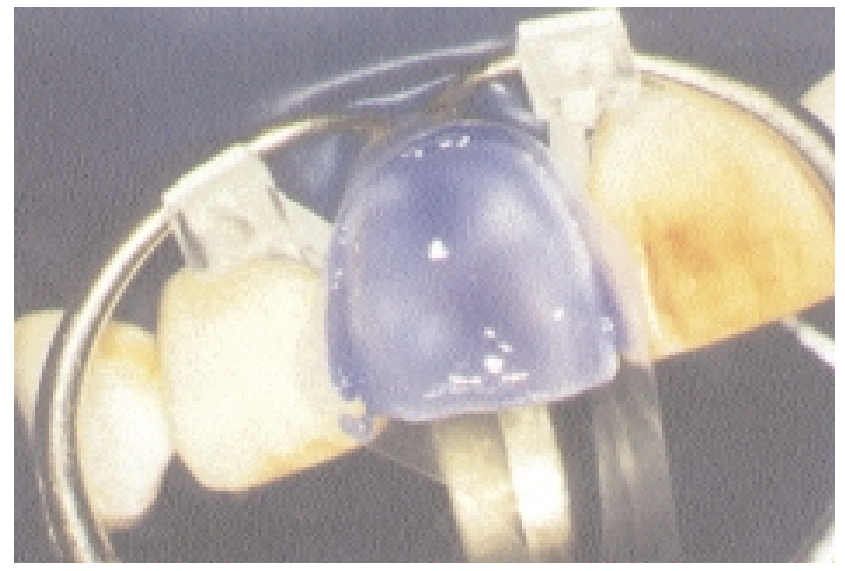

Figura 10: Acondicionamiento de la superficie vestibular del diente 11.

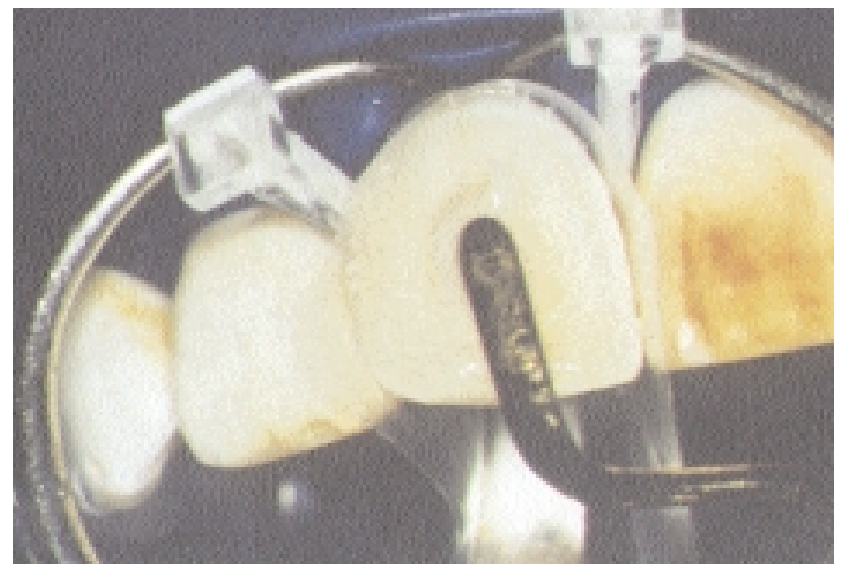

Fig.12: Inserción y moldeado de la resina.

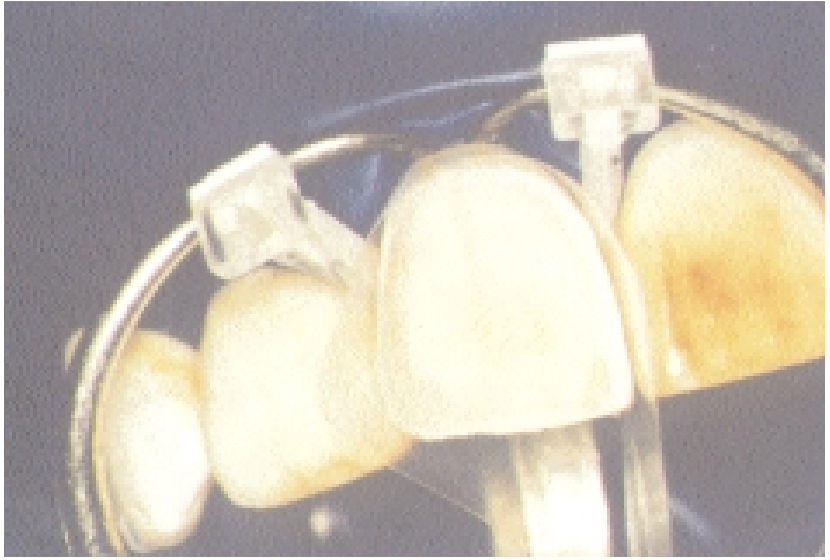

Figura 9: Colocación de Contour Strip 2 y cuña deflectora.

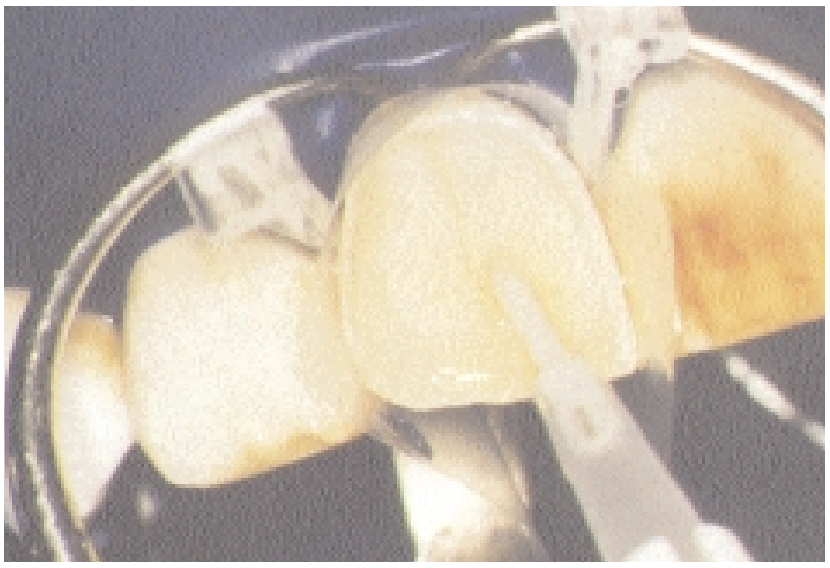

Figura 11: Se aplica sistema adhesivo al esmalte acondicionado, se insufla una suave corriente de aire y se fotopolimeriza.

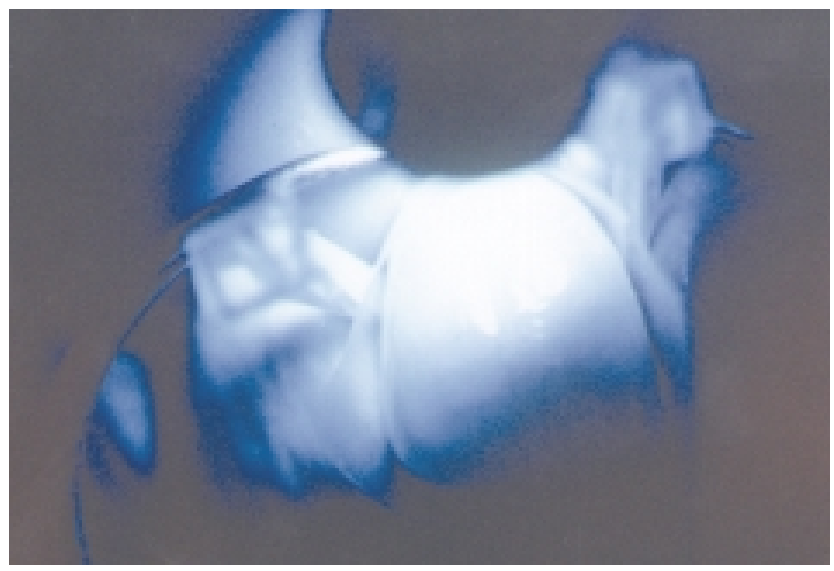

Figura 13: Fotopolimerización del material.

RCOE, 2003, Vol 8, №4, 415-421 
Se adapta y coloca una tira gingival de acetato, Contour Strip $2^{\mathrm{TM}}{ }^{\mathrm{M}}$ (Vivadent), además de la cuña deflectora $^{14}$, Luci Wedge ${ }^{\circledR}$ (Hawe), con la finalidad de evitar que el ácido, sistema adhesivo y material de obturación, afecten a los elementos dentarios vecinos (fig. 9).

Se acondiciona el esmalte de la superficie vestibular del elemento con ácido fosfórico al 37\% para luego, aplicar el sistema de adhesivo y fotopolimerizar (figs. 10, 11).

Se procede a la inserción del material de obturación, que en este caso, al tener muy poco espesor la preparación y para evitar una sobreobturación en volumen, se utilizó una primera capa de resina compuesta fotopolimerizable microhíbrida, y moldeándola, se fotopolimerizó. Se empleó entre

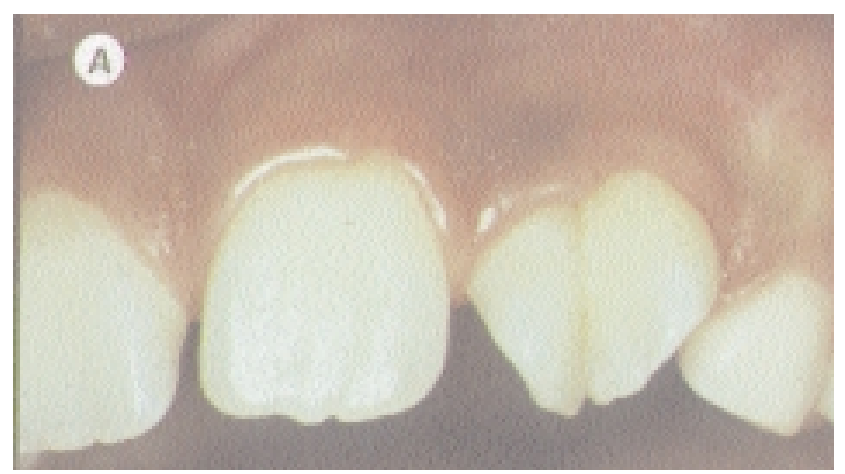

Figura 15 A: Diente 22 con geminación.

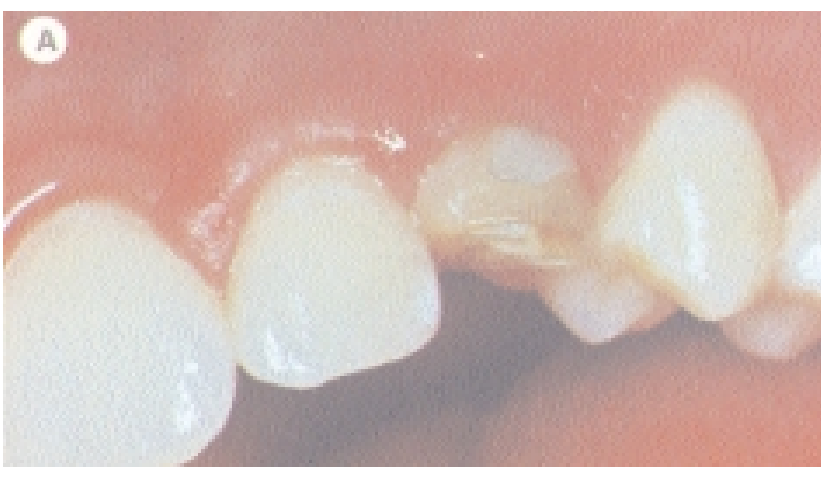

Figura 16 A: Canino superior izquierdo temporal, afectado. capas un tinte de color blanco, para las caracterizaciones de los elementos dentarios tratados, y se terminó de cubrir la cara vestibular con una resina

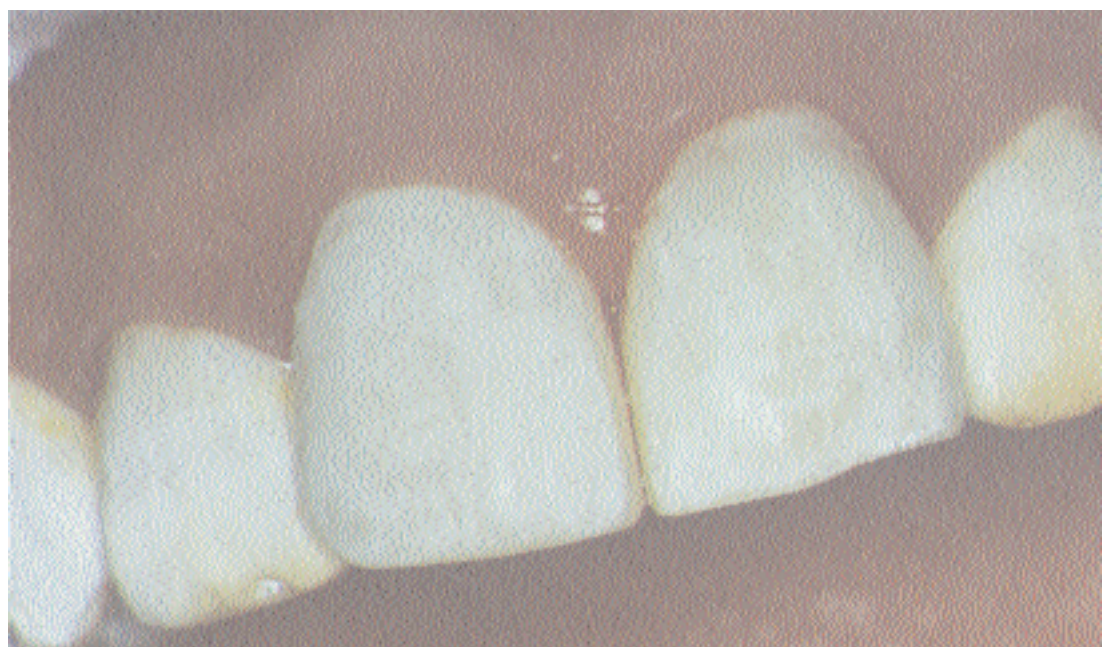

Figura 14: Carillas directas de resinas compuestas en los dientes 11 y 21 terminadas. compuesta fotopolimerizable de micropartículas (figs. 12, 13).

Se recomienda colocar el tinte entre capas de resina compuesta, ya

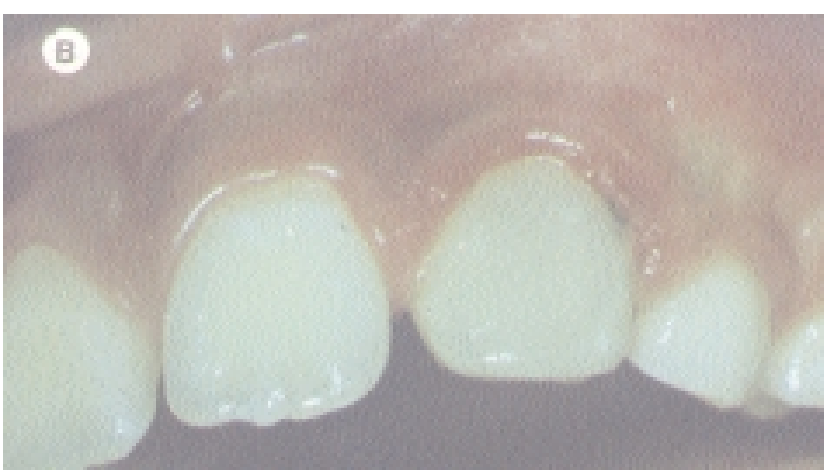

Figura 15 B: Solución clínica con carilla directa.

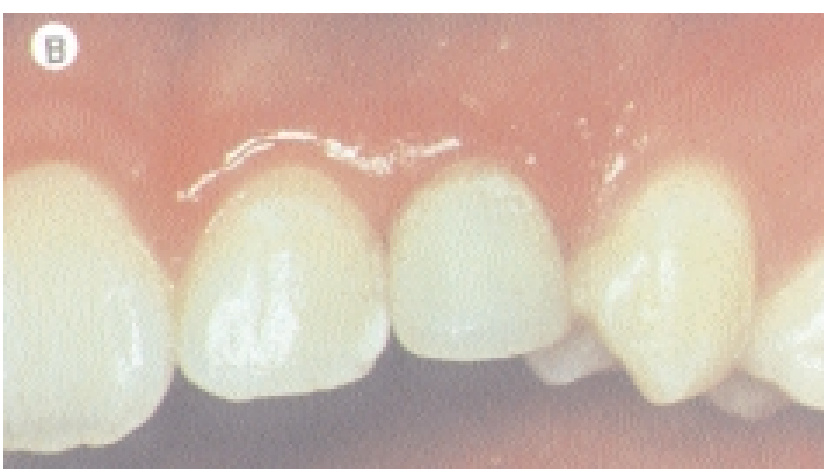

Figura 16 B: Solución clínica con carilla directa.

RCOE, 2003, Vol 8, №4, 415-421 
que al ser de baja viscosidad, se puede desprender fácilmente de la superficie de la restauración ${ }^{10^{* *}}$. Una vez polimerizada la última capa de resina compuesta, se realiza el terminado, pulido y control de la restauración (fig. 14).

Al finalizar la restauración, se recomienda al paciente no ingerir bebidas o alimentos con colorantes, como té, café, mate, como así también, evitar fumar ${ }^{10^{* *}, 11^{*}}$.

Otras de las aplicaciones clínicas de este tipo de restauración, pueden ser, para solucionar casos de elementos geminados, como en situaciones especiales, transformando un ele- mento temporal en permanente (figs. 15 A y $B, 16$ A y B).

\section{Conclusiones}

Esta técnica se emplea para casos clínicos que presentan distintas alteraciones en la superficie vestibular del elemento dentario, resolviéndose con un sistema de resinas compuestas fotopolimerizables en forma directa y con un bajo costo. Es necesario tener, además del conocimiento científico, el sentido artístico para lograr éxito en este tipo restauraciones.
La principal duda que se nos plantea, es su duración, ya que, la resina compuesta es susceptible de decoloración, lo que disminuye el resultado estético a largo plazo; pero creemos, que es importante mantener las estructuras dentarias naturales sanas hasta tanto sea necesario realizar otro tipo de restauración y mientras tanto se obtiene mayor tiempo útil de los elementos dentarios involucrados, debiéndose realizar controles periódicos clínicos-radiográficos, para posteriormente, observar su comportamiento en la boca a través de los años.

\section{Bibliografía recomendada}

Para profundizar en la lectura de este tema, el/los autor/es considera/an interesantes los artículos que aparecen señalados del siguiente modo: *de interés $* *$ de especial interés.

1. Cuello JL, Solla CJ, Pittaro E, Iglesias C. Estética Conservadora en Operatoria Dental. Rev Asoc Odontol Argent 1998;86:288-91.

2. Peumans M, Van Meerbeek B, Lambrechts P, Vuylsteke-Wauters M, Vanherle G. Resultados Clínicos de Carillas de Porcelana: evaluación de cinco años. Quintessence (ed. esp.) $1999 ; 12: 227-37$

3. Simonsen RS, Calamia JR. Tensile bond strenght of etched porcelain (resumen 1154). J Dent Res 1983;62:297.

4. Horn RH. Porcelain laminate veneers bonded to etched enamel. Dent Clin North Am 1983;27:671-84

5. Macchi R. Materiales Dentales. Restauraciones Indirectas. Buenos Aires: Panamericana, 2000.

6. Goldstein RE. Estética Odontológica. Buenos Aires: Intermédica, 1980
7. Bhaskar SN. Patología Bucal. Buenos Aires: El Ateneo, 1977

8. Kohen S, Aberstain E, Bergolis E, Capurro M. Blanqueamiento en Dientes Vitales. Rev Asoc Odontol Argent 1992;80:106-11.

9. Di Bella G. Blanqueamiento Dentario. Rev Asoc Odontol Argent 1998;86:288-91.

10**. Baratieri LN et al. Restauraciones Adhesivas Directas en Dientes Anteriores Fracturados. Sao Paulo: Santos Livraria, 1998.

El autor despierta el interés clínico y orienta en

forma racional el protocolo clínico de las carillas directas con resinas compuestas.

11*. Barrancos Mooney J. Operatoria Dental. Buenos Aires: Panamericana, 1999.

Establece las consideraciones terapéuticas a tener en cuenta en la técnica de blanqueamiento y restauraciones estéticas directas.
12. Carvalho MM, Babá MF, Machado A, Morais CV. Facetas Laminadas de Porcelana: Caso Clínico. Rev Odontol Univ Santo Amaro 1999;4:38-42.

13. Baratieri LN. Monteiro SJ, Andrada MAC, Arcari GM. Carillas de Composite: Una nueva técnica. Quintessence (ed. esp.) 1992;7:14-20.

14. Cuello JL. Influencia de la Cuña Deflectora en la Polimerización, Microfiltración y Adaptación Marginal de Materiales Restauradores Estéticos Fotopolimerizables en Preparaciones por Tunelización Oblicua. Tesis Doctoral. Universidad Nacional de Córdoba. Facultad de Odontología. Córdoba. Argentina, 2000.

15. Jordan RE et al. Composites en Odontología Estética. Técnicas y Materiales. Barcelona: Salvat, 1987. 